\title{
Upper Lumbar Intradural Disc Herniation: A Rare Case Report and Etiologic Analysis
}

\author{
Jia Chen $\mathbb{D}^{1,2}$ \\ Hai-Jian $\mathrm{Ni}^{1,2}$ \\ Fei Xue ${ }^{3}$ \\ Yun-Shan Fan ${ }^{1,2}$ \\ Xi-Fan $\mathrm{Li}^{4}$ \\ Xian-Zhen Chen $^{3}$ \\ Shi-Sheng $\mathrm{He}^{1,2}$ \\ 'Department of Orthopaedics, Shanghai \\ Tenth People's Hospital, Tongii University \\ School of Medicine, Shanghai, 200072, \\ People's Republic of China; ${ }^{2}$ Spinal Pain \\ Research Institute, Tongji University \\ School of Medicine, Shanghai, 200072, \\ People's Republic of China; ${ }^{3}$ Department \\ of Neurosurgery, Shanghai Tenth People's \\ Hospital, Tongji University School of \\ Medicine, Shanghai, 200072, People's \\ Republic of China; ${ }^{4}$ Department of \\ Radiology, Shanghai Tenth People's \\ Hospital, Tongji University School of \\ Medicine, Shanghai, 200072, People's \\ Republic of China
}

Correspondence: Shi-Sheng He Spinal Pain Research Institute, Tongji University School of Medicine, 30I Yanchang Middle Road, Jing'an District, Shanghai, 200072, People's Republic of China

Email tjhss74I8@tongji.edu.cn

Xian-Zhen Chen

Department of Neurosurgery, Shanghai

Tenth People's Hospital, Tongji University

School of Medicine, 30I Yanchang Middle

Road, Jing'an District, Shanghai, 200072,

People's Republic of China

Email chenxianzheny@।26.com
Background: Intradural disc herniation (IDH) is a rare type of disc degeneration that infrequently affects the upper lumbar spine. Pre- and intraoperative diagnosis and surgical management of IDH are challenging. The present case study provides insight into these aspects of upper lumbar IDH and discusses possible mechanisms.

Case Description: A 63-year-old female with a history of chronic lower back and leg pain presented with an acute lumbar sprain that had occurred 1 month prior. The pain progressed and spread to the front of the left thigh, which affected her ability to lift her leg when ascending/descending stairs. Sagittal gadolinium-enhanced magnetic resonance imaging (MRI) revealed a disc protruding into the ventral dural sac showing a hawk-beak sign, and the posterior edge of the disc annulus and local posterior longitudinal ligament was broken. Total L2 laminectomy was performed, and the dorsal side of the dural sac was exposed and incised to enable exploration of the ventral side of the dura. We found two free fragments protruding into the inner wall of the dura through the left ventral dura mater defect. After carefully and completely removing the mass, we repaired the defect and performed internal fixation. Postoperative pathologic analysis confirmed that the mass was nucleus pulposus tissue from the degenerated disc. The patient's pain significantly improved after surgery, and she was able to walk normally at the 1-month follow-up.

Conclusion: Upper lumbar IDH is an extremely rare type of disc degeneration. An enhanced MRI scan can provide diagnostic evidence, but the final diagnosis requires surgical exploration of the path of herniation and pathologic examination of the mass lesion.

Keywords: lower back pain, radiating pain, upper lumbar, intradural disc herniation, case report

\section{Introduction}

Lumbar disc herniation is one of the most common causes of acute or chronic lower back pain. ${ }^{1}$ Lumbar intradural disc herniation (IDH) is a rare type of disc degeneration accounting for $0.04-0.33 \%$ of all disc herniations. ${ }^{2}$ IDH affects the upper lumbar (L1/2/3/4) less frequently than the lower lumbar (L4/5 and L5/S1). ${ }^{3-5}$ Patients with chronic lower back pain often have upper lumbar IDH caused by acute lumbar sprain from heavy manual work. ${ }^{6,7}$ Preoperative radiographic findings can be misinterpreted as intradural tumors, cysts, or metastases, ${ }^{8}$ making definitive preoperative diagnoses difficult. ${ }^{5}$ Most cases rely on surgery for the final diagnosis, but surgical management of IDH - especially in the upper lumbar region - is also challenging, with a high risk of nerve injury. ${ }^{9}$ This case study describes the clinical diagnosis and surgical treatment of a patient with L2/3 upper lumbar IDH and discusses possible pathogenic mechanisms. The case is reported in accordance with SCARE criteria. $^{10}$ 


\section{Case Description}

A 63-year-old female with a history of chronic lower back and leg pain presented at our outpatient clinic with an acute lumbar sprain that had occurred 1 month prior. The patient described progressive lumbar pain radiating to the front of the left thigh that necessitated the use of crutches for walking and ascending/descending stairs. Prior to coming to our clinic, the patient had no pain relief with nonsteroidal anti-inflammatory drugs. There was no family history and no bladder or bowel symptoms. Neither physical therapy nor epidural steroid injections relieved the pain (visual analog scale score of 8 on a scale of 0 to 10). Neurologic examination revealed decreased pinprick sensation on the anterior aspect of the left thigh. The left-side femoral nerve stretch test was positive. Her knee jerk reflex was decreased in the left lower extremity. Muscle testing revealed reduced iliopsoas muscle strength.

A lateral lumbar spine X-ray revealed lumbar degeneration and osteophyte hyperplasia at the edge of vertebral body. Dynamic lumbar spine X-ray indicated instability between the L2 and L3 segments (Figure 1A). Sagittal T1- and T2weighted magnetic resonance imaging (MRI) showed rupture of the posterior disc fibrous annulus and loss of continuity of the posterior longitudinal ligament (PLL) at the L2/3 level (Figure 2A and B). Sagittal gadolinium-enhanced MRI showed large disc protrusion into the ventral dural sac, which appeared as a hawk-beak sign (Figure 2C). The same was observed on axial MRI in which the PLL appeared torn in the left posterior direction, with a large mass squeezing the dura mater (Figure 3A). It also showed peripheral ring enhancement of the sequestered herniated disc (Figure 3B).

IDH was highly suspected but the possibility of tumor/ cyst could not be ruled out; therefore, prompt surgical intervention was critical. The operation was jointly performed by experienced senior orthopedic surgeons (S.-S.H., H.-J.N., and J.C.) and neurosurgeons (X.-Z.C. and F.X.). Intraoperative durotomy was selected to fully examine the lesion and reduce the risk of postoperative dural tearing or nerve injury. Under general anesthesia, we made a posterior midline incision and performed total decompressive laminectomy at the L2 segment. With the aid of a surgical microscope, we carefully detached and resected the ligamentum flavum to uncover the dura mater in the spinal canal. The dura was swollen, hard, and immobile. A close inspection of the extradural space from the left side did not reveal any free protrusions, but the ventral dural sac was tightly adhered to the PLL. We performed durotomy according to the preoperative plan, and nerve rootlets were squeezed out of the dorsal dural incision (Figure 4A). After identifying the nerve structure, the cauda equina nerve was gently pulled to the right side. From the left dorsolateral view, we observed a smooth
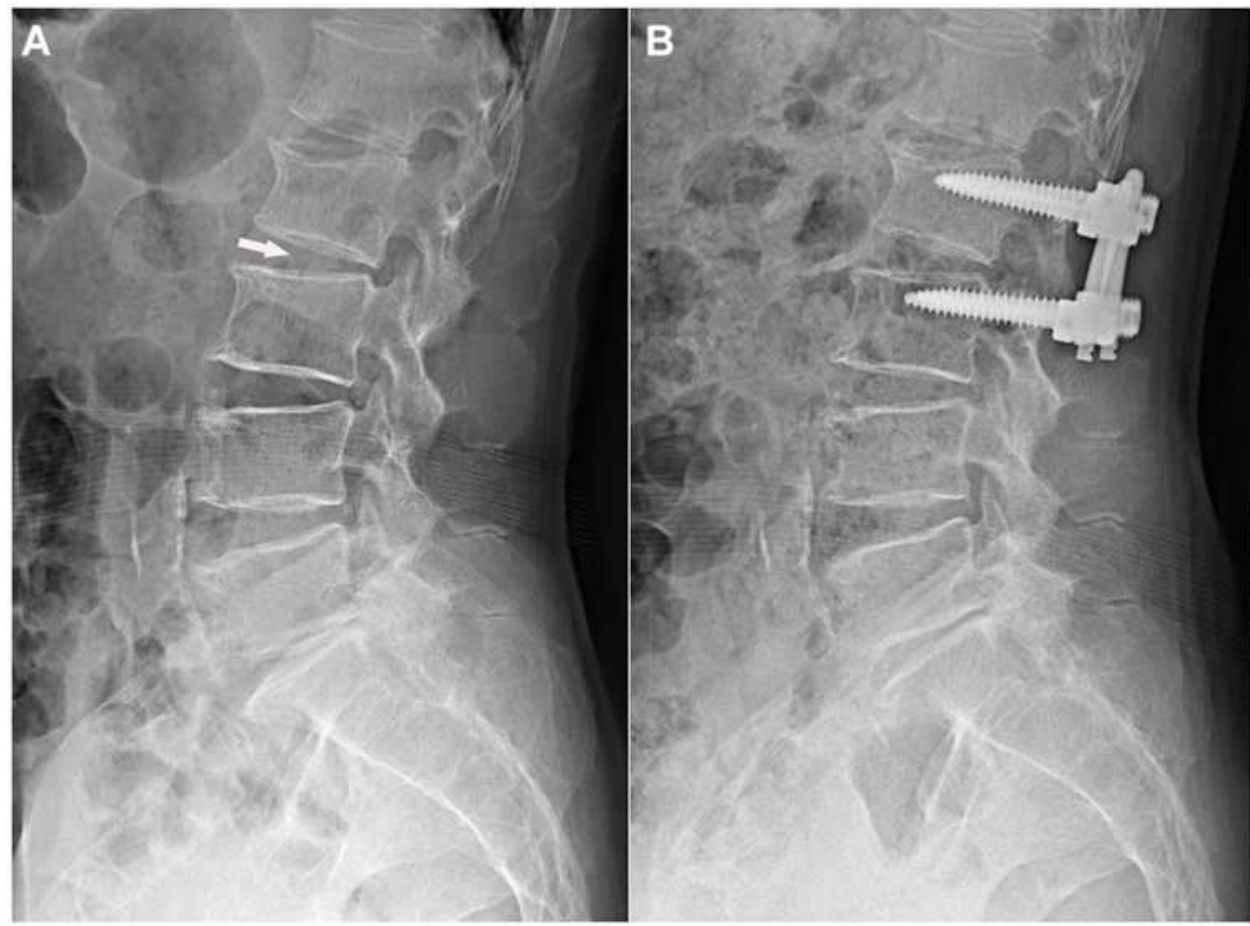

Figure I Preoperative radiographs showing lumbar degeneration and instability between the L2/3 segments (A, white arrow) and internal fixation to stabilize the local intervertebral space (B). 

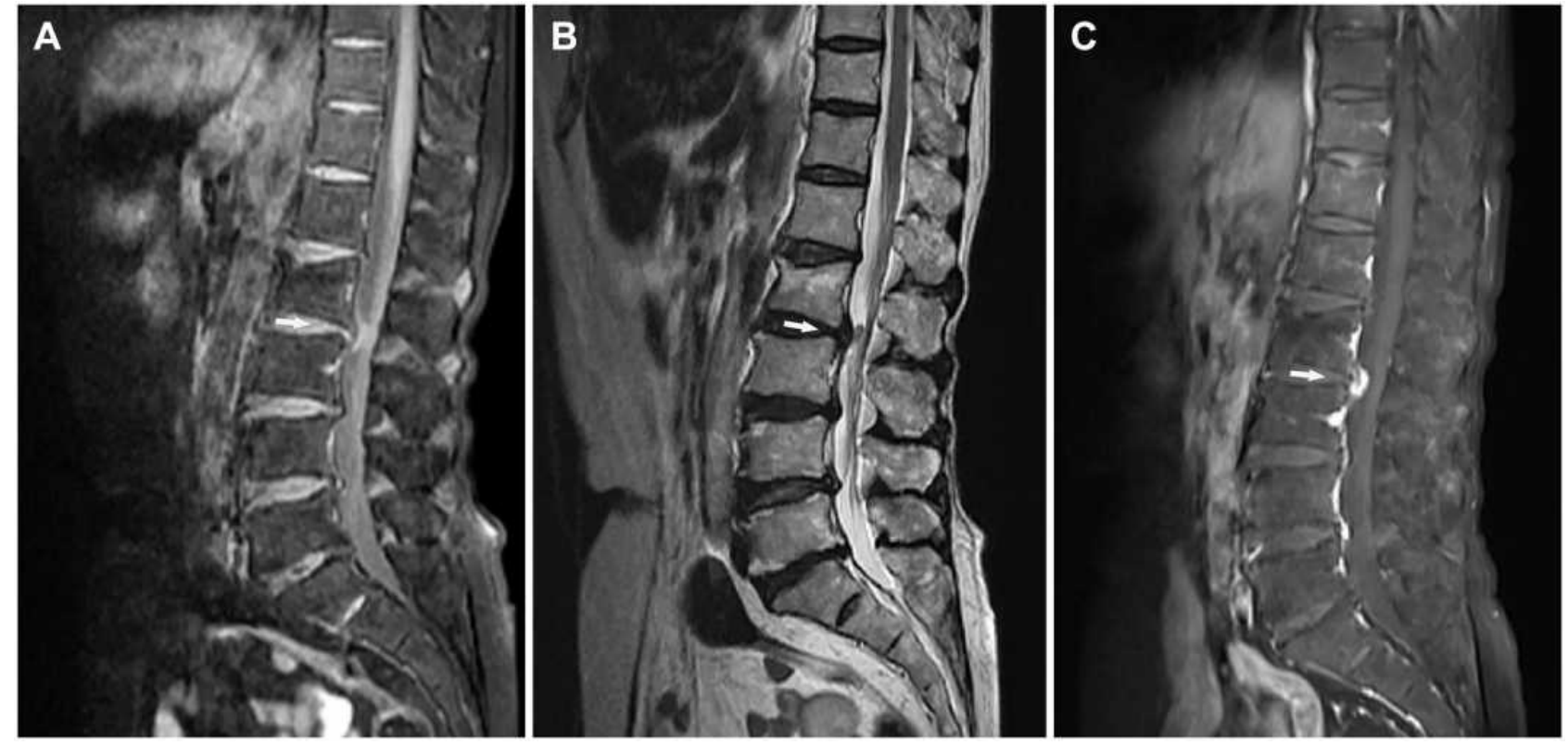

Figure 2 Sagittal TI-weighted (A) and T2-weighted (B) MR images showing posterior disc fibrous annulus rupture and loss of PLL continuity at the L2/3 level. (C) Enhanced MR image showing a "hawk-beak" sign. White arrows indicate disc herniation and associated radiographic changes.
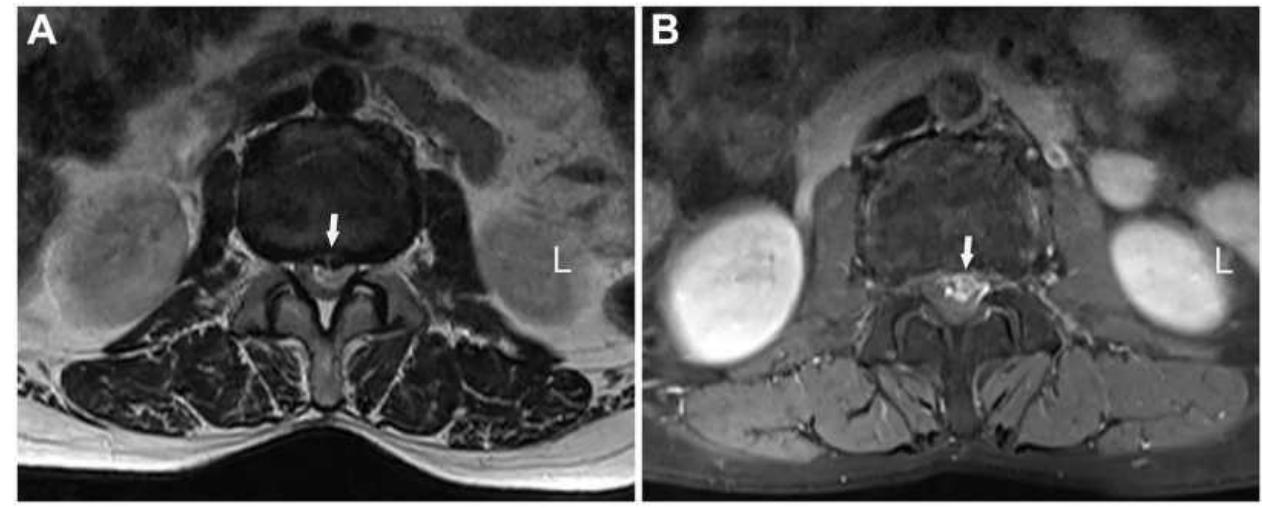

Figure 3 (A) Axial T2-weighted MR image showing the torn PLL (A, white arrow).

(B) Enhanced MR image showing a ring sign (B, white arrow).

surface with hard fragments lodged in the inner wall of the ventral dura (Figure 4B). After extirpating two free fragments with sizes of approximately $1.7 \times 0.8 \times 0.4$ and $1.0 \times 0.5 \times 0.4 \mathrm{~cm}^{3}$ (Figure 4D), the cauda equina nerve relaxed and the dura mater slackened. We found a thinned round defect ( $\sim 8 \mathrm{~mm}$ in diameter) within the ventral dura (Figure $4 \mathrm{C})$. From the left ventrolateral field of view, we found that the defect adhered to the PLL and was difficult to move. No free or sequestered fragments were found on the other sides of the intradural extra-arachnoid space. The ventral defect was repaired using a dura-allograft, and the dorsal dura was closed with continuous sutures. Internal fixation was used to stabilize the local intervertebral space (Figure 1B).
Histopathologic examination of the extruded disc-like material at $50 \times$ and $100 \times$ magnification revealed that the mass contained degenerated fibrocartilage, nucleus pulposus tissue, and hyperplastic inflammatory granulation tissue (Figure 5A-C). The patient's pain was significantly alleviated after 2 weeks of following postoperative instructions regarding incision care, pain medication, and rehabilitation. There were no postoperative complications or adverse events such as cerebrospinal fluid (CSF) leakage or wound infection. We evaluated the patient's compliance with the recommended rehabilitation exercises using a questionnaire and found that she had followed the instructions. At the 1-month follow-up, the patient was asymptomatic. 


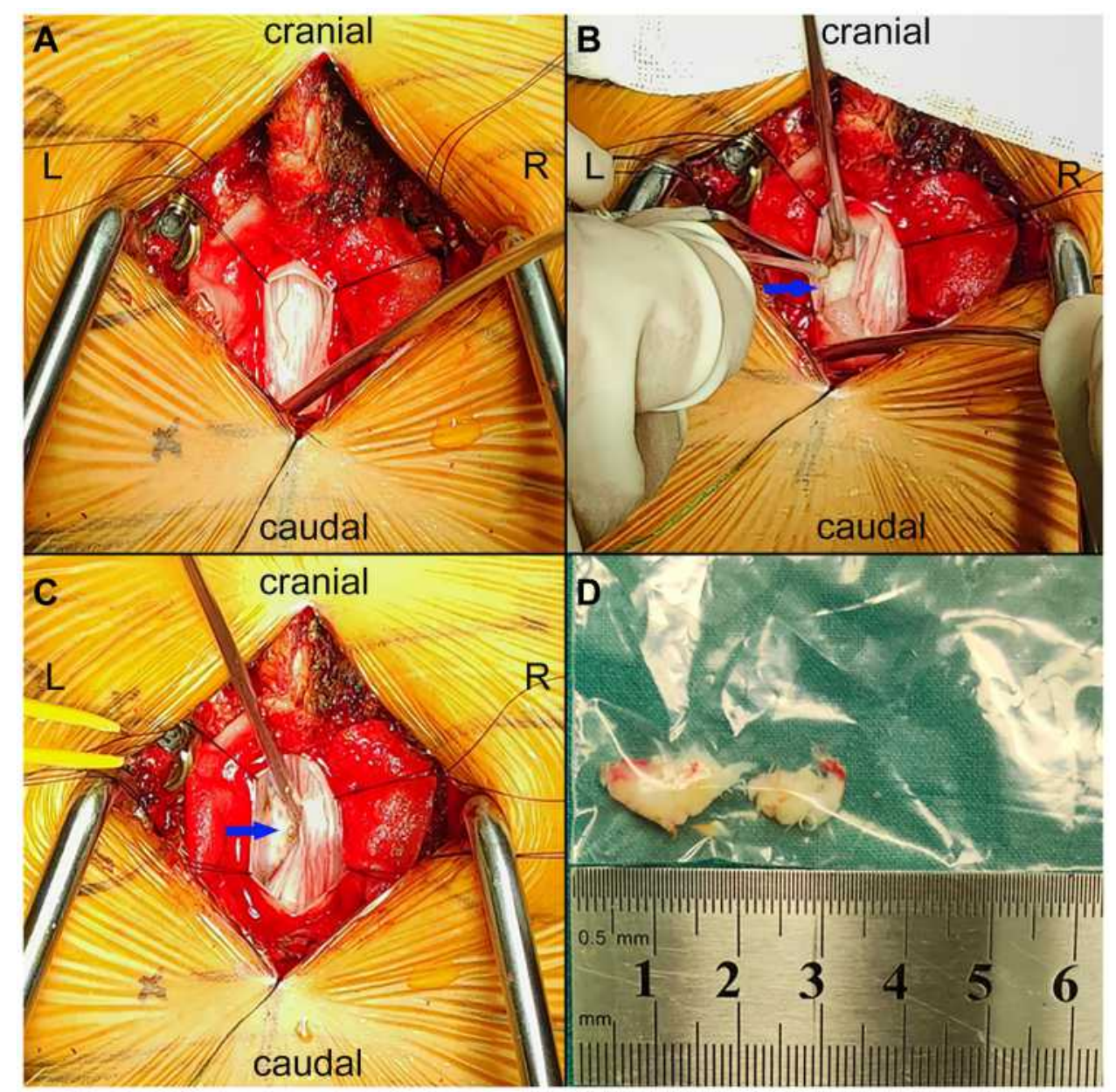

Figure 4 (A) A midline durotomy was performed. (B) Smooth surface with hard fragments lodged in the inner wall of the ventral dura (blue arrow). (C) Thinned round defect within the ventral dura (blue arrow). (D) Image showing the sizes of the two herniated disc fragments.
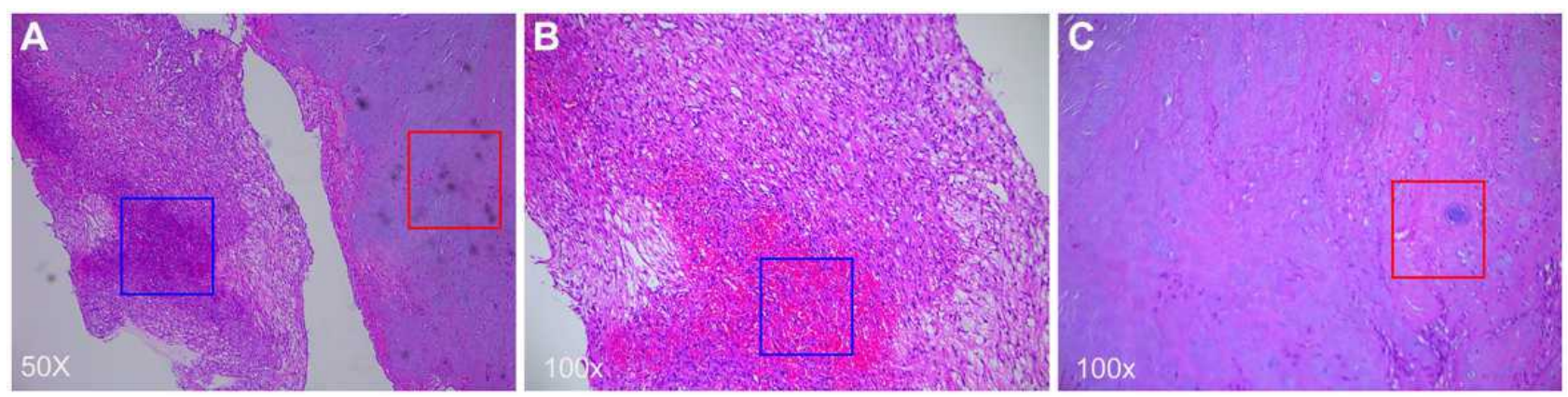

Figure 5 (A) Degenerated fibrocartilage (red box) and hyperplastic inflammatory granulation tissue (blue box) on histopathologic examination (50x magnification). (B) Proliferation of small blood vessels and fibroblasts accompanied by inflammatory cell infiltration (blue box, I00x magnification). (C) Degenerated fibrocartilage and nucleus pulposus tissue (red box, 100× magnification).

\section{Discussion}

IDH at the L2/3 level is extremely rare. ${ }^{11}$ The exact pathophysiologic mechanism is unclear, but congenital or acquired dense adhesions between the PLL and ventral dural sac are thought to be involved. ${ }^{11,12}$ To our knowledge, IDH is primarily influenced by acquired predisposing factors such as chronic inflammation, degenerative disease, iatrogenic conditions, trauma, local infection, and hemorrhage. ${ }^{13-15}$ These etiopathogenic factors can result in local adhesions and facilitate migration of 
extruded disc material into the intradural sac space. Patients who have an intraoperative diagnosis of upper lumber IDH frequently exhibit intermittent neurogenic symptoms for months/years and are referred to the hospital upon experiencing acute onset of back pain with or without neurological deficits. ${ }^{16,17}$

A herniated disc combined with annulus fibrosus rupture can lead to the formation of a local "fingertip" structure that squeezes and firmly affixes the PLL to the dural sac. Accompanied by frequent lumbar movement-especially in the upper lumbar spine which has greater mobility than in the lower lumbar segments - the fingertip structure can repeatedly press the PLL and dura mater to promote adhesion. ${ }^{18}$ A ruptured disc spontaneously releases inflammatory factors that stimulate and erode local adhesions. ${ }^{4,19}$ This erosion coupled with the mechanical squeezing force exerted by herniated disc fragments causes thinning of the adhered local dural sac ${ }^{18}$ and its eventual rupture, resulting in the entry of free disc fragments into the subdural space where they compress and stimulate the cauda equina nerve. ${ }^{6,20}$ At this point, patients with chronic intermittent pain may experience acute and more serious neurologic symptoms. ${ }^{16}$ Our patient had a history of chronic lower back and leg pain resulting from a lumbar sprain that progressed to serious acute pain, which was highly suspected to be caused by disc degeneration.

Thoroughly analyzing a patient's medical history, physical examination, and imaging are essential for preoperative diagnosis of IDH. However, a definitive diagnosis based on imaging features is challenging for several reasons. ${ }^{8,21}$ First, the characteristic findings reported in the literature are not present in every case. ${ }^{17,22}$ Nonetheless, IDH is highly suspected when the "hawk beak" sign, Y sign, ring enhancement, or "crumble disc" sign are observed on preoperative radiologic examination. A report of two cases of lumbar IDH described two representative MRI findings that aroused suspicion of extruded disc herniation: abrupt loss of PLL continuity in the sagittal MR image indicating annulus fibrosus rupture and a sharp beak-like pattern (known as the "hawk beak" sign) in the axial $\mathrm{T} 2$-weighted image. ${ }^{20}$ These imaging features can aid preoperative diagnosis and safe intraoperative planning.

In another study, preoperative MRI revealed free herniated disc fragments perforating the ventral dural sheath, separating the dura and arachnoid in a Y-shaped pattern (ie, the $\mathrm{Y}$ sign). ${ }^{22}$ The ring sign on gadolinium-enhanced
MRI occurs when contrast medium is visible at the periphery of the subdural disc fragment. ${ }^{23}$ However, unlike chronic inflammation, acute extruded disc herniation shows no ring-like contrast enhancement, ${ }^{24}$ which originates from the condensation of contrast medium in the surrounding granulation tissue where inflammation stimulates angiogenesis, resulting in homogeneous contrast enhancement of the mass. ${ }^{25}$ The "crumble disc" sign reflects free disc debris that has entered the thecal sac. Although not very common, it is nonetheless a highly specific imaging feature that can be used for the preoperative diagnosis of IDH. ${ }^{26}$ Some studies have also suggested that gas-filled herniation on computed tomography (CT) or MRI is evidence of intradural extension of disc fragments. ${ }^{27,28}$ In our study, T1- and T2-weighted axial MRI showed posterior disc fibrous annulus rupture and loss of PLL continuity at the L2/3 level. Gadoliniumenhanced MRI revealed peripheral ring enhancement of the sequestered herniated disc on axial images, while the "hawk beak" sign in sagittal images was enhanced by the contrast medium, leading us to suspect IDH. However, MRI did not show signs of gas-filled herniation in our case.

Secondly, even with neuroimaging advances, it remains difficult to clearly differentiate IDH from other spinal pathologies such as benign tumor, arachnoiditis, hematoma, meningeal cyst, and metastasis. ${ }^{8,25,29,30}$ Lumbar puncture can be performed to evaluate spinal pressure, and cytologic/molecular markers in CSF can be examined for differential diagnosis of IDH.

Thirdly, it is difficult to distinguish whether a mass lesion is sequestered extradurally, intradurally, or in the extra-arachnoid space. This is because from a radiologic perspective, the sequestered disc itself and other confounding elements such as perfused CSF and local dense adhesions or cysts may also be visible on MRI.

When patients manifest severe neurologic symptoms and IDH is highly suspected based on radiologic evidence, immediate surgical intervention should be considered as the first-line treatment. ${ }^{11}$ The surgical procedure involves careful exploration and meticulous dissection to avoid neurologic injury. ${ }^{9}$ Although intradural herniation has been examined by transforaminal posterolateral endoscopy, the main surgical approach is durotomy. ${ }^{31}$ The choice of partial or total laminectomy for decompression should be based on preoperative imaging findings. The surgical procedure should include palpating the tension of the dura and searching for remnant disc fragments in 
the epidural and intradural space, as well as the adhesion area and adjacent caudal and cranial areas. Once the dorsal dural sac is opened, the arachnoid mater and nerve rootlets should be gently pulled to one side to allow examination of the intradural mass lesion and expose the ventral defect. It is highly recommended that a surgical microscope is used to facilitate clear visualization of the surgical area and reduce the risk of nerve rootlet injury. ${ }^{5}$ At the end of surgery, it is important to properly repair both the dorsal and ventral dura defects to minimize the risk of postoperative CSF leakage. Some surgeons have used hemostatic material or autogenous fascial or fat grafts instead of continuous sutures to close the ventral defect without CSF leakage or other complications. ${ }^{9,11,25}$ Spinal fusion and internal fixation are also recommended to stabilize the local intervertebral space and avoid secondary disc herniation. ${ }^{9,32}$

Compared to previous reports, the novelty of the present case study is that it provides strategies for preoperative imaging diagnosis of upper IDH and a paradigm for the surgical diagnosis of this condition including selection of the surgical approach and intraoperative examination. We also described aspects of the operation that require special attention. Our case study thus provides surgeons with important information for IDH patient management and operative planning.

\section{Conclusion}

Upper lumbar IDH is an extremely rare type of disc degeneration; possible causes and pathophysiologic mechanisms include congenital or acquired dense adhesions between the posterior disc fibrous annulus, PLL, and ventral dural sac. Adhesion causes thinning of the dural sac and promotes herniated disc protrusion into the sac. Preoperative enhanced MRI can reveal specific imaging features that facilitate diagnosis. Once IDH is highly suspected based on radiologic evidence, prompt surgical intervention should be considered as the first treatment option to ensure rapid recovery.

\section{Abbreviations}

CSF, cerebrospinal fluid; CT, computed tomography; IDH, intradural disc herniation; MRI, magnetic resonance imaging; PLL, posterior longitudinal ligament.

\section{Patient Perspective}

After the operation, I found that I can walk up and down stairs without crutches. Now I can return to a normal life. I'm willing to share my case with other doctors.

\section{Ethics Approval}

This study was approved by the ethics committee of Shanghai Tenth People's Hospital (Approval no. 20K163).

\section{Informed Consent}

Written informed consent was obtained from the patient for publication of this case report and accompanying images.

\section{Disclosure}

The authors report no conflicts of interest in this work.

\section{References}

1. Dunn KM, Croft PR. Epidemiology and natural history of low back pain. Eura Medicophys. 2004;40:9-13.

2. Kataoka O, Nishibayashi Y, Sho T. Intradural lumbar disc herniation. Report of three cases with a review of the literature. Spine. 1989;14:529-533. doi:10.1097/00007632-198905000-00011

3. D'Andrea G, Trillò G, Roperto R, et al. Intradural lumbar disc herniations: the role of MRI in preoperative diagnosis and review of the literature. Neurosurg Rev. 2004;27:75-80; discussion 81-82. doi:10.1007/s10143-003-0296-3

4. Han IH, Kim KS, Jin BH. Intradural lumbar disc herniations associated with epidural adhesion: report of two cases. $J$ Korean Neurosurg Soc. 2009;46:168-171. doi:10.3340/jkns.2009.46.2.168

5. Ducati LG, Silva MV, Brandão MM, Romero FR, Zanini MA. Intradural lumbar disc herniation: report of five cases with literature review. Eur Spine J. 2013;22(Suppl 3):S404-S408. doi:10.1007/s00586-012-2516-4

6. Tempel Z, Zhu X, McDowell MM, Agarwal N, Monaco ER. Severe intradural lumbar disc herniation with cranially oriented free fragment migration. World Neurosurg. 2016;92:582.e1-582.e4. doi:10.1016/j.wneu.2016.06.024

7. Kim HS, Eun JP, Park JS. Intradural migration of a sequestrated lumbar disc fragment masquerading as a spinal intradural tumor. $J$ Korean Neurosurg Soc. 2012;52:156-158. doi:10.3340/ jkns.2012.52.2.156

8. Kim JS, Lee SH, Arbatti NJ. Dorsal extradural lumbar disc herniation causing cauda equina syndrome: a case report and review of literature. J Korean Neurosurg Soc. 2010;47:217-220. doi:10.3340/ jkns.2010.47.3.217

9. Pedaballe AR, Mallepally AR, Tandon V, Sharma A, Chhabra HS. An unusual case of transdural herniation of a lumbar intervertebral disc: diagnostic and surgical challenges. World Neurosurg. 2019;128:385-389. doi:10.1016/j.wneu.2019.05.103

10. Agha RA, Fowler AJ, Saeta A, et al. The SCARE statement: consensus-based surgical case report guidelines. Int $J$ Surg. 2016;34:180-186. doi:10.1016/j.ijsu.2016.08.014

11. Moon SJ, Han MS, Lee GJ, et al. Unexpected intradural lumbar disk herniation found during transforaminal endoscopic surgery. World Neurosurg. 2020;134:540-543. doi:10.1016/j.wneu.2019.11.121 
12. Yildizhan A, Paşaoğlu A, Okten T, et al. Intradural disc herniations pathogenesis, clinical picture, diagnosis and treatment. Acta Neurochir. 1991;110:160-165. doi:10.1007/BF01400685

13. McCormick PC, Stein BM. Miscellaneous intradural pathology. Neurosurg Clin N Am. 1990;1:687-699. doi:10.1016/S10423680(18)30796-4

14. Fiorenza V, Ascanio F, Di Lorenzo I. Clinicopathologic features of thoracolumbar interdural disc herniations: a retrospective case series with a systematic literature review. World Neurosurg. 2020;139: e391-e398. doi:10.1016/j.wneu.2020.04.015

15. Theodorou DJ, Theodorou SJ, Kakitsubata Y, Papanastasiou EI, Gelalis ID. Posterior and anterior epidural and intradural migration of the sequestered intervertebral disc: three cases and review of the literature. J Spinal Cord Med. 2020;1-6. doi:10.1080/ 10790268.2020.1730110

16. Ponzo G, Furnari M, Umana GE, et al. Intradural lumbar disc herniations at the L1-L2 level: a case study and literature review. Surg Neurol Int. 2020;11:67. doi:10.25259/SNI_108_2020

17. Low J, Rowland D, Kareem H. L1/2 intradural disc herniation with compression of the proximal cauda equina nerves: a surgical challenge. World Neurosurg. 2020;142:147-151. doi:10.1016/j. wneu.2020.06.163

18. Floeth F, Herdmann J. Chronic dura erosion and intradural lumbar disc herniation: CT and MR imaging and intraoperative photographs of a transdural sequestrectomy. Eur Spine J. 2012;21(Suppl 4):S453S457. doi:10.1007/s00586-011-2073-2

19. Cunha C, Silva AJ, Pereira P, et al. The inflammatory response in the regression of lumbar disc herniation. Arthritis Res Ther. 2018;20:251. doi:10.1186/s13075-018-1743-4

20. Choi JY, Lee WS, Sung KH. Intradural lumbar disc herniation - is it predictable preoperatively? A report of two cases. Spine J. 2007;7:111-117. doi:10.1016/j.spinee.2006.02.025

21. Thohar AM, Ikbar KN, Brilliantika SP, et al. Challenges in intradural disc herniation diagnosis and surgery: a case report. Ann Med Surg. 2020;58:156-159. doi:10.1016/j.amsu.2020.08.022
22. Sasaji T, Horaguchi K, Yamada N, Iwai K. The specific sagittal magnetic resonance imaging of intradural extra-arachnoid lumbar disc herniation. Case Rep Med. 2012;2012:383451. doi:10.1155/ 2012/383451

23. Wasserstrom R, Mamourian AC, Black JF, Lehman RA. Intradural lumbar disk fragment with ring enhancement on MR. AJNR Am $J$ Neuroradiol. 1993;14:401-404.

24. Mailleux R, Redant C, Milbouw G. MR diagnosis of transdural disc herniation causing cauda equine syndrome. J Belg Soc Radiol. 2006;89:303-305.

25. Liu CC, Huang CT, Lin CM, Liu KN. Intradural disc herniation at L5 level mimicking an intradural spinal tumor. Eur Spine J. 2011;20 (Suppl 2):S326-S329. doi:10.1007/s00586-011-1772-Z

26. Mailleux P, Marneffe V, Michel I, Dehullu JP. The "Crumble disc sign": a specific MRI sign of intradural lumbar disc herniation, allowing a preoperative diagnosis. J Belg Soc Radiol. 2015;99:25-29.

27. Nam KH, Han IH, Cho WH, Choi BK. A pure fluid-filled intradural cyst associated with intradural disc herniation and possible pathogenesis: a case report. Spine J. 2013;13:e11-e15. doi:10.1016/j.spinee.2013.06.009

28. Sei A, Mizutamari M, Fujimoto T, Taniwaki T, Mizuta H. Gas-filled intradural cysts of the lumbar spine and the possible pathogenesis. Spine J. 2009;9:e6-e8. doi:10.1016/j.spinee.2009.01.010

29. Kim DS, Lee JK, Jang JW, et al. Clinical features and treatments of upper lumbar disc herniations. $J$ Korean Neurosurg Soc. 2010;48:119-124. doi:10.3340/jkns.2010.48.2.119

30. Rodrigo V, Claramonte M, Martín M, Calatayud JB. Intradural disc herniation: How I do it. Acta Neurochir. 2018;160:945-947. doi:10.1007/s00701-018-3510-3

31. Kim HS, Pradhan RL, Adsul N, et al. Transforaminal endoscopic excision of intradural lumbar disk herniation and dural repair. World Neurosurg. 2018;119:163-167. doi:10.1016/j.wneu.2018.07.244

32. Tamaki Y, Sakai T, Miyagi R, et al. Intradural lumbar disc herniation after percutaneous endoscopic lumbar discectomy: case report. J Neurosurg Spine. 2015;23:336-339. doi:10.3171/2014.12. SPINE14682
Journal of Pain Research

\section{Publish your work in this journal}

The Journal of Pain Research is an international, peer reviewed, open access, online journal that welcomes laboratory and clinical findings in the fields of pain research and the prevention and management of pain. Original research, reviews, symposium reports, hypothesis formation and commentaries are all considered for publication. The manuscript management system is completely online and includes a very quick and fair peer-review system, which is all easy to use. Visit http:// www.dovepress.com/testimonials.php to read real quotes from published authors. 